\title{
Asymmetry and Overdetermination in Swain's Counterfactual Theory of Causation
}

\author{
CIIRISTOPIJER HITCHCOCK \\ Universily of Pittsburgh
}

Hume's second definition of causation described effects as being counterfactually dependent upon their causes: one 'object' caused another "where, if the first object had nut been, the sccond never had existed."I This definition lay dormant for more than two centuries before it was revived and given its best known formulation by David Lewis.2 Several years ago, however, an alternative analysis of causation using counterfactuals was suggested by Marshall Swain.3 I wish to examine Swain's account critically as a polential alternative to Lewis's.

Swain's analysis begins with some important definitions: 4

(D1) The proposition that if $A$ were true, then $C$ would be true is true at a world $w$ iff:

either (1) there are no possible $A$-worlds

or (2) some $A$-world where $C$ holds is closer to $w$ than is any $A$-world where $C$ does not hold.

(D3) Where $c$ and $e$ are occurrent events, e depends counterfactually on $c$ iff: if $c$ had not occurred, then $e$ would not have occurred.

(D4) $c$ and $e$ are distinct events iff:

(1) $c$ is not identical to $e$; and

(2) neither event is a constituent of the other. 5

(D5) Where $c$ and $e$ are occurrent events, e depends causally on $c$ iff:

(1) $c$ and $e$ are distinct events; and

(2) if $c$ had not occurred, then $e$ would not have occurred.

1 David Hume, An Enquiry Concerning Iluman Understanding, Section VII.

2 David Lewis, "Causation," Journal of Philosophy 70 (1973): 556-67.

3 Marshall Swain, "A Counterfactual Analysis of Event Causation," Philosophical Studies 34 (1978): 1-19. Swain's recent position is essentially no different from that presented in the original article.

4 llbid., pp. 3-5.

5 Swain gives a more detailed account of event distinction in "Causation and Distinct Events," in Peter van Inwagen, ed., Time and Cause, (Durdrecht: Reidel, 1980)), 155-169. 
(D6) Where $c, d_{1}, \ldots, d_{n} e$ is a sequence of occurrent events (but not necessarily a temporal sequence, and where $c$ and $e$ may be the only members), this scquence is a causal chain iff:

(1) $d_{1}$ depends causally on $c$; and

(2) $d_{2}$ depends causally on $d_{1}$; and...

(n) $d_{n}$ depends causally on $d_{n-1}$; and

$(n+1)$ e depends causally on $d_{n}$.

Swain suggests the following as a first approximation to an analysis of causation:

(D7) Where $c$ and $e$ are specific events that occurred, $c$ is a cause of $e$ iff: there is a causal chain of occurrent events from $c$ to $e$.

He argues, however, that (D7) is inadequate for two reasons. The first is that it does not guarantee the asymmetry of causation. If $c$ is a cause of $e$, then $e$ can not be a cause of $c$. (D7) seems to violate this principle. If $e$ had not occurred, then some cause of $e$ must also have failed to occur. If $c$ is the cause which would fail to occur, there will be a chain of causal dependence from $e$ to $c$; so according 10 (D7), $c$ caused $e$ and $e$ caused $c$. Lewis argues that the relation of counterfactual dependence is asymmetric, and that causal asymmetry follows as a corollary. According to Lewis, the closest possible world in which e does not occur will be one in which all of the causes of $e$ occul anyway. In his paper 'Counterfactual Dependence and Time's Arrow,'6 Lewis offers criteria for judging of two worlds $w_{1}$ and $w_{2}$, which is closer to the actual world $w$. His criteria, however, do not guarantec that $w_{1}$ will be closer to $w$ than is $w_{2}$ if $w_{2}$ violates the laws of $w$ and $w_{1}$ does not. Thus, the closet possible world in which a particular event $e$ does not occur may (and typically will) be one in which the laws of nature of the actual world are violated. Swain rejects this view of counterfactuals; he argues that the candidates for being the possible world closest to $w$ will be ones in which the natural laws of $w$ are not violated.7 As a result, Swain's relation of counterfactual dependence will not, in general, be asymmetric. Swain tries to capture the asymmetry of causation by imposing two additional conditions on definition (D7): 8

(D7) Where $c$ and $e$ are specific events that occurred, $c$ is causally prior to $e$ iff:

(i) there is a causal chain of occurrent cvents from $c$ to $e$;

6 Lewis, "Counterfactual Dependence and Time"s Arrow," Noùs 13 (1979): 455-476.

7 See Swain, "A Counterfactual Analysis," pp. 7-10.

4 Swain, "A Counterfactual Analysis," p. 11. 
(ii) where $w_{1}$ is a world in which coccurs and e does not occur, and $w$ is the actual world, $w_{1}$ would only have to have been different from $w$ in the following respect: some event $a$ (other than c) which occurs in $w$ and upon which e depends causally in $w$ fails to occur in $w_{1}$ :

(iii) where $w_{2}$ is a world in which e occurs and $c$ does not occur, and $w$ is the actual world, $w_{2}$ would have to be different from $w$ in at least the following respects: (1) some event $f$ (other than e) which occurs in $w$ and upon which $c$ depends causally in $w$ fails to occur in $w_{2}$; and (2) some event $g$ occurs in $w_{2}$ such that $e$ is not causally dependent upon $g$ in $w$ but $e$ is causally dependent upon $g$ in $w_{2} .9$

Informally, $c$ can be a cause of $e$, but not vice versa, if there is a world in which $c$ occurred, but e didn't, which is closer to the actual world than is any in which e occurred and $c$ didn't, where the distance between two worlds is measured by the number of events that occur in one world but not both. ${ }^{10}$ This is intended to rule out the possibility of $c$ and $e$ being treated as causes of one another.

The second problem with (D7), according to Swain, is that it will fail in cases of causal overdetermination: cases where two (or more) events, cach one sufficient by itself to cause $e$, both occur, each having an equal claim to being a cause of $e$. It is not true of any ovendetermining cause, that had it not occurred, the effect would not have occurred. An example would be a man's being killed by being simultancously shot through the heart by two bullets: had either bullet not been shot, he still would have died. Causal overdetermination must not be confused with causal preemption. In cases of causal preemption, iwo (or more) events occur, each sufficient by itself to cause $e$, but where the occurrence of one prevents the others from aclually having a hand in bringing about the effect. (D7) is equipped to treat cases of causal preemption, "but in cases of overdetermination, it runs into trouble. Swain's strategy for tackling the problem of

\footnotetext{
9 A printing error in the original article caused the last clause to misread: "some event $g$ occurs in $w 2$ such that $e$ is not causally dependent upon $f$ in $w$ but $e$ is causally dependent upon $f$ in $w_{2} . "$

10 In this informal paraphrase, I ignore difficultics in interpreting the modal expressions "would have to be different" and "would only have to have been different". Since definition (D7") already involves quantification over possible worlds, it is to be hoped that these modal expressions can be eliminated in favor of quantified expressions. Swain's intentions are not entircly clear on this point, however.

"Sec, c.g. Lewis, "Causation," p. 191; Swain, "A Counterfactual Analysis," pp. 13-4.
} 
overdetermination rests on the following observation. Suppose two events, $c$ and $d$, causally overdetermine the event $e$. In the actual world, neither $c$ nor $d$ is causally prior to $e$. If $d$ had not occurred, however, $c$ would have been causally prior to $e$ by virtuc of a mechanism or process which artually occurred. This is in contrast with the case where $c$ is precmpted by $d:$ in this case, $c$, in d's absence, would have been causally prior to $e$ by virtue of a mechanism which did not actually occur. Swain's account of overdetermination transforms this observation into an alternative set of conditions sufficient for two events to be related as cause and effect. Adding his account of overdetermination to his account of asymmetry yields Swairis full account of causation:12

(D7") Where $c$ and $e$ are specific events that occurred, $c$ was a cause of $e$ iff:

Either (A) (i), (ii), and (iii), as above in (D7); Or

(B) Some set of events $D=\left(d_{1}, d_{2} \ldots, d_{n}\right)$ occurred (possibly having only one member) such that

(a) If $c$ had not occurred, and if any member $d_{i}$ of $D$ had occurred, but no other members of $D$ had occurned, and if $e$ had occurred anyway, then there would have been a causal chain from $d_{i}$ to $e$ consisting wholly of occurrent events, and $d_{i}$ would have been causally prior to e; and

(b) If no member of $D$ had occurred, and if $c$ and $e$ had occurred anyway, then there would have been a causal chain from $c$ to $e$ consisting wholly of occurnent events, and $c$ would have been causally prior to $e$.

Unfortunately, the sceming advantages of Swain's revisions to (D7) vanish under careful examination. I will present several examples where Swain's analysis fails to correctly diagnose the causal situation. ${ }^{13}$ Some wil! exploit technical details in Swain's account. It will be argued, however, that the difficulties raised by these examples can not be circumvented by severing the relevant details from the rest of the account. Other examples will point to more general difficulties with Swain's analysis.

12Swain, "A Counterfactual Analysis," p. 16.

13 It deserves to be mentioned that Martin Bunzl ("Causal Preemption and Counterfactuals," Philosophical Studies 37 (1980): 115-124) and Wayne Davis ("Swain's Counterfactual Analysis of Causation," Philosophical Studies 38 (1980): 169-176) have suggested counterexamples to Swain's account. I believe these counterexamples may be handled by straightforward and natural revisions, but I will not discuss this issue here. 
The first telling problem is that according to Swain's analysis, there are no causes; at any rate, the existence of causes implies a restriction on possible worlds which seems unwarranted. As a preliminary, note that while condition (D7") is disjunctive, disjunct $(B)$ is parasitic upon the condition described in disjunct (A). The clause " $c$ would have been causally prior to $e^{\prime \prime}$ in $\left(D 7^{\prime \prime}\right)(B)(b)$ entails that there is some possible world $w$ (not necessarily the actual world) in which $c$ and $e$ satisfy the relation of causal priority defined in (D7') and repeated in (D7")(A). It follows that if $c$ does not stand in the relation of causal priority to $e$ in any possible world, then both disjuncts of (D7") will fail.

Now suppose that $c$ and $e$ are any two occurrent events-we may suppose, to make the counterexample more vivid, that $c$ actually causes $e$. If $c$ is a cause of $e$, according to Swain's analysis, there must be some possible world in which $c$ is causally prior to $e$. That is, there must be some world, $w$, with respect to which $c$ and $e$ satisfy condition (DT). Clause (iii) of (D7) asserts: "where $w_{2}$ is a world in which e occurs, and $c$ does not occur,... $w_{2}$ would have to be different from $w$ in at least the following |respect|:...some event $g$ occurs in $w_{2}$ such that $e$ is not causally dependent upon $g$ in $w$ but $e$ is causally dependent upon $g$ in $w 2$." It is easy, however, to construct a world in which e occurs and $c$ doesn't, which does not have such an additional causal dependence. Let $w_{2}$ be the world which differs from $w$ in these respects: 1) $c$ fails to occur in $w$; and 2) $e$ is brought about by (wo overdetermining causes (say, $d$ and $f$, neither one of which occurs in $w$. (Sce diagram 1.)

\section{Diagram 1}

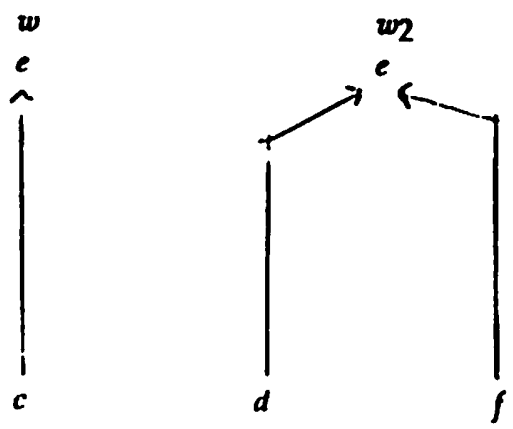

Since the events $d$ and foverdetermine e, e is causally dependent on neither of then, so condition (D7')(iii) fails. (Recall that causal dependence, as defined in (D5), requires counterfactual dependence, which is absent in case's of causal overdetermination.) There is a similar counterexample if $e$ is brought alxuut by a pre-empting cause in w2.

No argument has been offered for the conclusion that w2 is a possible world, but it is hard to sce what might disqualify it. Swain allows that an 
effect may be brought about by different causes in different possible workds, and he admits that an effect may be brought about by overdetermining causes. $w_{2}$ simply combines these two possibilities.

One possible solution to this problem immediately suggests itself: allow for the possibility of disjunctive events. In the example above, $d$-or-f could be considered as a single event. If this event had not occurred-if neither $d$ nor $/$ had occurred-in $w_{2}$, then e would not have occurred. With this ontological addition the counterexample vanishes. This would patch up the problem, but it would raise a new problem: Swain would owe an account of disjunctive events. Simply admitting disjunctive events without some modification of the theory would lead to undesirable results. For example, suppose my striking the cue ball on a particular occasion caused the sinking of the eight ball: Then Swain's current account would treat the disjunctive event of my-striking-the-cue-ball-or-a-nuclear-holocaust as a cause of the eight ball's going into the side pocket; this disjunctive event would also satisfy Swain's criterion (D7). This is certainly odd: had the disjunctive event occurred by virtuc of the occurrence of the second disjunctthe nuclear holocaust--the cight ball would have disintegrated rather than have gone into the side pocket. Thus, if Swain is to modify his account by admitting disjunctive events, he will have to make many further revisions. Ad hominem, Swain's choice to treat causal overdetermination by adding disjunct (B) to (DT'), rather than by admitting disjunctive events, shows an unwillingness to make these revisions.

There are, of course, many more revisions to (D7') which might be attempted. The problems uncovered by the above counterexample, however, are deep enough to raise doubts about the possibility of any successful revision. It may well be that the only revision of (D7')(iii)(2) that will save the causal phenomena is the replacement of "e is causally dependent upon $g$ in $w_{2}$ " with " $c$ is caused by $g$ in $w_{2}$ " or some extensional equivalent. It seems at least prima facie likely that in order for $e$ to be brought about in the absence of one of its actual (non-overdetermining) causes, it would be both necessary and sufficient to have some event be a new cause of it. Of course, to make this modification would be to firt with vicious circularity. Swain himself foreshadows this difficulty:

...to imagine $e$ without $c$, you need to imagine that something prevented $c$ from occurring and something else caused $e$, but to get $c$ without $e$ you only need to imagine that something prevented $c$ from causing $e$. Of course, this way of putting the matter neatly begs the question concerning the causal facts in this case, but the difference noted provides us with the key to capluring asymmetry. ${ }^{14}$

14 Swain, "A Counterfactual Analysis," p. 11. 
Our counterexample involving an overdetermined event in one possible world satisfies Swain's informal, circular critcrion for $c$ to be a cause of $e$, but not his formal, nen-circular one. This suggests that the circularity is not so casily avoided.

I wish to discuss one final flaw which Swain's account of causal asymmetry shares with any counterfactual analysis of causation which disqualifies worlds which violate the actual laws of nature when evaluating counterfactuals. Such accounts rob counterfactual theories of their ability to treat cases of causal preemption. Suppose, for instance, that $c$ caused $e$ via $d$, preempting the causal chain from f to $e$ via g. (See diagram 2.)

\section{Diagram 2}

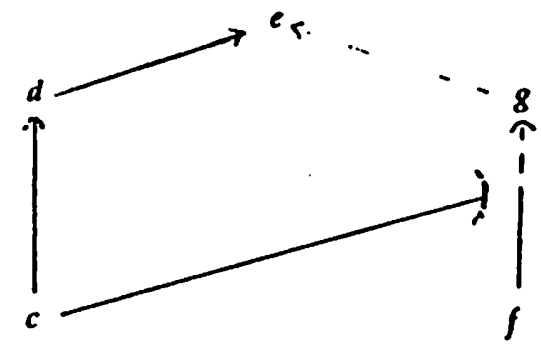

If $c$ had not occurred, $f$ would have caused $g$, which would have caused $e$. As it happened, however, $c$ prevented $g$ from occurring, thus breaking the link from $f$ to $e$. The success of Swain's account depends on there being a chain of counterfactual dependence from $c$ to $e$. It is not true that if $c$ had not occurred, then $e$ would not have occurred; it is alleged, however, that if $c$ had not occurred, then $d$ would not have occurred, and that had $d$ not occurred, e would not have occurred (the causal chain from $f$ to $e$ having already been preempted due to $c$ 's preventing 8 ). The success of the latter counterfactual, however, demands that the counterfactual 'If $d$ had not occurred, $c$ would have occurred anyway' be true: a dangerous assumption if the actual laws of nature must hold in the closest possible world in which $d$ fails to occur. Some cause of $d$ must fail to occur in this closest possible world, and there is no guarantce that $c$ will not be this disappearing cause.

Pushing the above problems aside, we may now focus our attention on the second disjunct of (D7"), which gives Swain's account of overdetermination. There is a serious flaw with this account: it grants preempted cause's the status of genuine causes. Suppose that $d$ caused $e$, but that $c$ would have caused $e$ had $d$ not occurred. (See diagram 3.) 
Diagram 3

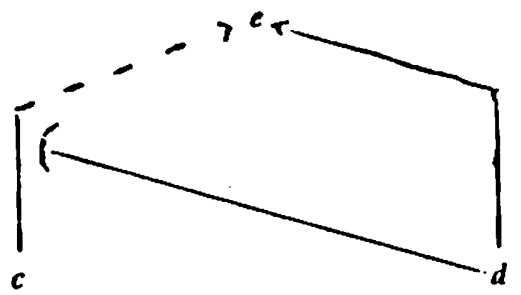

On close inspection, we see that $c$ and $e$ satisfy disjunct (B) of (DT"):

Consider the set $D=[d]$.

(a) If $c$ had not occurred, and if $d$ (the only member of $D$ ) had occurred, and if $e$ had occurred anyway, then there would have been a causal chain from $d$ to $e$ consisting wholly of occurrent events, and $d$ would have been causally prior to e. This follows directly from the hypothesis the $d$ did, in fact, cause $e$.

(b) If no member of $D$ had occurred, and if $c$ and $e$ had occurred anyway, then (i) there would have been a causal chain from $c$ to $e$ consisting wholly of occurrent events, and (ii) $c$ would have been causally prior to $e$. Clause (ii) is satisficd by hypothesis: if $d$ had not occurred, $c$ would have caused $e$.

Swain evidently wants to prevent the analysis from going through by saying that (b)(i) is not satisfied: since $c$ was, in actuality, preempled, there is no causal chain from $c$ to e consisting wholly of occurrent events. Here Swain would be simply mistaken. There is such a causal chain, namely the sequence $c$, $e$ : in the absence of $d, e$ is causally dependent upon $c$, and both $c$ and $e$ are occurnent events. The fact that in the absence of $d$, the events leading from $c$ to $e$ did not all occur in the actual world does not affect the status of the causal chain c, e. Clearly, Swain's notion of a causal chain consisting wholly of occurrent events is a much weaker one than it first appeared: it does not come close to the intuitive notion of an occurrent causal mechanism or process which motivated Swain's account of causal overdetermination. While Swain's strategy is intuitively comprehensible, it is hard to see how it can be explicated in Swain's vocabulary of causal chains. 15

15 Peter Menzics ("Probabilistic Causation and Causal Processes: A Critique of Lewis," Philosophy of Science 56 (1989): 642-663) offers an objection to Lewis's account which is analogous to that raised in this paragraph. 
I share Swain's discomfort with Lewis's counterfactual theory of causation. Swain's goal, a counterfactual theory of causation in which the actual laws of nature are held sacred when evaluating the possible world closest to the actual one, is an admirable one. The failure of his account to provide a sound alternative to Lewis's, however, suggests that Swain's admirable goal is also an impossible one. 16

\section{References}

Bunz.l, Martin (1980). "Causal Preemption and Counterfactuals." Philosophical Sludies 37: 115-124.

Davis, Wayne (1980). "Swain's Counterfactual Analysis of Event Causation." Philosophical Studies 38: 169-76.

Hume, David (1748). An Enquiry Concerning Humen Understanding.

Lewis, David (1973). "Causation." Journal of Philosophy 70' 556-67.

13: $455-476$.

(1979). "Counterfactual Dependence and Time's Arrow." Nols

Menzies, Peter (1989). "Probabilistic Causation and Causal Processes: A Critique of Lewis." Philosophy of Science 56: 642-663.

Swain, Marshall (1978). "A Counterfactual Analysis of Event Causation." Philosophical Sludies 34: 1-19.

(1980). "Causation and Distinct Events," in Peter van Inwagen, ed., Time and Cause (Dordrecht: Reidel), pp. 155-169.

16 This material is based upon work supported under a National Science Foundation Graduate Fellowship; I am extremely grateful for this support. I would like to thank Danicl $M$. Hausman for his guidance and many helpful comments upon carlier drafts. 